\title{
A METHOD FOR ROAD LIGHTING AUDIT AND SAFETY SCREENING AT URBAN INTERSECTIONS
}

Matin S. Nabavi Niaki (Corresponding author)

Masters Student, Department of Civil, Geological and Mining Engineering

Polytechnique Montréal

65 Rue Sherbrooke Est, Apt 619

Montréal (Québec) Canada H2X 1C4

Phone: +1 (416) 270-3702

Email: matin.nabaviniaki@polymtl.ca

\section{Nicolas Saunier}

Ph.D. ing., Associate professor

Department of Civil, Geological and Mining Engineering

Polytechnique Montréal, C.P. 6079, succ. Centre-Ville

Montréal (Québec) Canada H3C 3A7

Phone: +1 (514) 340-4711 ext. 4962

Email: nicolas.saunier@polymtl.ca

\section{Luis F. Miranda-Moreno}

Ph.D., Assistant Professor

Department of Civil Engineering and Applied Mechanics

McGill University

Room 268, Macdonald Engineering Building, 817 Sherbrooke Street West

Montréal (Québec) Canada H3A 2K6

Phone: +1 (514) 398-6589

Fax: +1 (514) 398-7361

Email: luis.miranda-moreno@mcgill.ca

\section{Luis Amador}

Ph.D., Assistant Professor

Department of Building, Civil and Environmental Engineering

Concordia University

Room EV-6.139, 1515 St. Catherine West

Montréal (Québec) Canada H3G 2W1

Phone: +1 (514) 848-2424 ext. 5783

Fax: +1 (514) 848-7965

Email: amador@encs.concordia.ca

\section{Jean-François Bruneau}

M. Sc., Adjunct Professor

Department of Applied Geomatics

Université de Sherbrooke

Room A4-250, FLSH, 2500 Blvd. de l'Université

Sherbrooke (Québec) Canada J1K 2R1

Phone: +1 (819) 821-8000 ext. 63217

Fax: +1 (819) 821-7944

Email: jean-francois.bruneau@usherbrooke.ca

Submitted to: Safety Data, Analysis and Evaluation (ANB20) committee for publication Word count: $6443+7$ tables and figures $(1750)=8193$

March 15, 2013 


\begin{abstract}
The review of recent research efforts in road lighting and safety shows an inconsistency in the methods to measure ambient road lighting. The importance of road lighting on improving night time safety is evident; however, the lack of actual illuminance field measurements results in a gap in the knowledge of whether installed road lighting provides adequate illuminance for clear visibility at night time or not. Previous studies considered the presence or absence of road lighting on safety without measuring actual illuminance of the road. This paper aims to propose a uniform methodology to perform a simple road lighting audit and safety screening that can be applied to any area.

To perform the proposed audit, a photometric sensor, data logger and information on the city lighting standards, geo-referenced accident data and traffic flow data are needed. To collect field measurements, the data collectors cross each side of the intersection with the sensors starting and ending $15 \mathrm{~m}$ before and after the intersection. Information on land use, road type, location of light poles, location of trees and weather conditions is collected. Based on the collected data, average illuminance of each approach of an intersection as well as the average illuminance of the whole intersection and the uniformity ratio of the intersection was calculated. These results are then used to compare to the city lighting standard to check if the installed road lighting is performing adequately. If illuminance values of an intersection were below the standard specifications, the intersections were ranked as sub-standard.

This methodology was then applied to a case study in Montréal, Québec, where $59 \%$ of the selected sample intersections had sub-standard lighting. Statistical analysis showed that the number of night time accidents was correlated to traffic flow (or the ratio of minor to major flows) and the fact that the intersection average intersection illuminance did not meet the standard. The factors contributing to average illuminance were clear sky, hour of the night of the data collection, and presence of light poles and commercial lights.
\end{abstract}




\section{INTRODUCTION}

The purpose of road lighting is to provide visibility, security and safety for all road users during the night (1-5). Once light poles are installed according to specification standards, they are assumed to provide adequate illumination to road users at night. However, lighting equipment might not be well maintained and there are hardly any follow-ups on the performance of lighting and its effect on safety. With time and resources constraints, there are usually few field measurements done by municipalities to check if lighting meets the specification standards. This is problematic because with the rapid change in traffic flow and land use, the amount of illumination needed for visibility also changes $(6,7)$. Road safety issues at night where lighting standards are not checked can be related to illumination deficiencies (1-4). Therefore, it is important to inspect the adequacy of road lighting performance on a regular schedule $(8,9)$.

A study done by the University of London reports that only a quarter of all travel done by cars are between the hours of $7 \mathrm{pm}$ and $8 \mathrm{am}$, yet this period accounts for $40 \%$ of fatal and serious injuries (9). Another European study confirms that even though only $25 \%$ of the vehicle-miles traveled is during the night, nearly $50 \%$ of fatalities occur in those hours, making night time fatality rate three times higher than the daytime rate (10). Night time travelling is therefore done a greater risk, but other important night time issues, not directly linked to lighting, such as drunk driving and speeding, along with infrequent police controls, also contributes to overall risk. This brings up the importance of investigating night time accidents factors, the most important being lack of clear visibility (1-4). Even though some studies looked at the effects of road lighting on accidents and safety $(1,3,11-16)$, a limited number of studies did actual field measurements. These studies use different ways of measuring illuminance most of which are cumbersome and often not sufficiently accurate (17). The methodology presented in this paper aims to propose a uniform practice to be applied to any area.

Studies considering road lighting and safety mostly measured only the presence and absence of light, or relied on before-after studies for road lighting implementations $(3,11,12,16$, 18-21). Although the presence or absence of lighting is a key factor, the amount of lighting provided could be inappropriate (too low or too high), resulting in non-adequate visibility and glare. More recent studies evaluate illuminance levels and meeting standards in relation with safety. One of the papers that considered illuminance measurements studied a sample of street lighting installations in urban areas using illuminance meters (20). The authors concluded that average road illuminance has a significant effect on road safety, and the effect propensity is higher at locations with more severe levels of injury. Another study aimed to find the relationship between crash injury severity at night time and average road lighting illuminance (21). Using illuminance measurements, they concluded that providing a certain amount of lighting in urban areas decreases the probability of crashes with injuries during night time.

This raises the question of the link between land use (residential, commercial, etc.), road lighting, and safety. If there is a link, what is the causal relationship between these factors? The assumption is that road lighting level is governed by land use development and therefore safety is related to the built environment and land use. For example, illuminance coming from other source than lighting poles is much higher in commercial areas.

This paper highlights the importance of conducting field audits on road lighting by introducing a simple method to systematically measure road lighting and evaluate its performance, and also by proposing a screening approach for identifying locations for lighting improvements. The methodology of auditing road lighting presented in this paper can be applied to any area to check if road lighting meets the requirements. A significant contribution with 
respect to past studies is to carry out actual field measurements of road lighting for analysis at urban and suburban intersections. The amount of ambient road light is measured using a photometric light sensor. Another goal of this paper is to study the relationship between lighting, safety and other characteristics of the built environment in urban and sub-urban areas. The methodology presented in this paper focuses on lighting at signalised intersections in urban and sub-urban areas as intersections are critical points in a road network where vehicles, pedestrians and cyclists share the same space. In Canada, more than $30 \%$ of fatalities and $40 \%$ of serious injuries occur at intersections (22).

The proposed road lighting audit methodology is applied to Montréal's signalized intersections to check if the cities road lighting specifications are met. A statistical analysis is also done to relate the number of night time accidents to average illuminance and built environment characteristics.

The remainder of the paper is organized as follows; the background section provides a brief review of earlier research in context. Followed by that is the proposed road lighting audit methodology. Further it is followed by the application of the methodology to a case study of urban and sub-urban signalized intersections in Montréal. Finally the paper is concluded and future work is discussed.

\section{BACKGROUND}

\section{Road Lighting}

The issue that people face after sunset is darkness and the lack of clear visibility. With the development of cities, the idea of illuminating human walkways during night time emerged. The main reason for illumination during night was to provide visibility, increase the sense of security and safety and to allow activities to take place in the later hours of the evening (23). Street lighting later on became a major factor in pedestrian safety and crime reduction during the night (23). With the rapid increase in population, vehicle ownership and size of cities, municipalities proposed lighting standard specifications aiming to create an environment with consistent lighting and adequate visibility for the safety of all road users. Yet there are no guarantees that the installed road lighting is performing sufficiently at all times, and therefore road lighting audits must be done to check if road lighting has an impact on safety or not.

In order to deal with light measurements, it is important to get familiar with units and technical terms. Illuminance is visible light as seen by the human eye and is measured in units of lux. The lux is carefully defined to weigh each wavelength by the luminosity function to reflect how light is perceived by human eyes (24). Average illuminance is the brightness of the road as seen by a driver. Uniformity ratio is a measure of how evenly lit the road surface is, and is calculated by dividing average illuminance by the minimum illuminance of the road segment.

\section{Road Lighting Specifications}

By its nature, a standard defines adequate and acceptable practices. Different countries, cities and municipalities follow different lighting specification standards $(8,12)$. Different standards require different pole heights, different distance between poles, different lamps and levels of lighting. The major North American lighting specification guide is the Illuminating Engineering Society of North America (IESNA) Lighting Handbook (6). The transportation association of Canada (TAC) also has a guide for the design of roadway lighting which provides lighting standards used by Canadian provincial transportation agencies (25). 
Most of the road lighting standards only present the average maintained illuminance levels for different types of intersecting roads (6). Some standards include a minimum illuminance and some give a maximum uniformity ratio value above which lighting will result in disability glare $(6,25)$.

\section{Road Safety}

In cities where the national/provincial and municipal road lighting are dealt with separately, road lighting throughout the city will not be consistent, and many roads may be under-lit (4). A major point of interest in a transportation network is therefore at intersections (26). Different municipalities may use different lighting standards, which may also be different from national/provincial lighting standards. This results in intersections where one street is lit according to provincial standards and the other street according to municipal standards. There are some evidence that if one street has a high average illuminance and the other has substantially lower light, a driver turning from the well-lit road to the under-lit street will take a few seconds for their eyes to adjust to the darker road and also, if the driver is turning from the under-lit road into the well-lit road, they will be blinded by light for the first few seconds (27-29). The glare recovery time ranges from 1 to 7 seconds depending on the age and optical health of the driver (30). Disability glare occurs when the introduction of a stray light source reduces one's ability to resolve spatial detail (30). The IES proposes maximum illuminance values and uniformity ratio to avoid the disability glare and the temporary reductions in visibility when the eye is adapting from alternately looking at areas of widely different illuminances (31). A study conducted by Box (31) found that the number of night time accidents decreased as light levels increased up to an illuminance threshold, and then increased for higher light levels, which is hypothesized to be related to the impact of glare in locations with substantial lighting variation.

Several studies have looked at road lighting and safety. These studies focused on the absence or presence of road lighting. For example, a study showed that the presence of road lighting at night not only reduces the risk of accidents, but also their severity (15). Another study conducted in the Netherlands showed that an improvement in the lighting from very bad to good in an urban area reduced accidents with injuries by approximately $30 \%(32,33)$. A study done in Minnesota looked at the effects of lighting on accident frequency for different intersection types, where the results showed that the presence of road lighting at intersections contributes to $12 \%$ lower night-to-day accident ratio with respect to an unlit intersections (1). Another study considering road lighting and safety concluded that the effect of road lighting on injury accidents during darkness is $49 \%$ on Dutch motorways (12). The same results were found in a study investigating the effect of lighting conditions on frequency and severity of road accidents at urban and rural roads in Greece. This research concluded that the presence of night time road lighting has an effect on improving traffic safety and reducing accident severity (11).

\section{ROAD LIGHTING AUDIT METHODOLOGY}

This section presents the step-by-step methodology to systematically perform a lighting audit at signalised and non-signalised intersections. This practice can be applied to any area if the required data and equipment are available. The main steps of data preparation and analysis are:

(1) Identifying data sources: obtaining the city lighting standards, accident data, and traffic flow.

(2) Data preparation: filtering accident data for accidents occurring at night time, and selecting intersections with night time accidents occurring in their vicinity. 
(3) Sample selection: identifying intersection hotspots based on traffic flow and the number of night time accidents.

(4) Data collection in the intersection sample: collecting illuminance and built environment characteristics in the selected sample intersections.

(5) Field data analysis: comparing average illuminance with standards and safety.

The following subsections will describe these steps in more details.

\section{Data Sources}

The first step is to find which lighting specification standards the city or municipality follows for installing road lighting. This information can be obtained from the city transportation department.

The primary type of data for this research is geo-referenced accident data, usually obtained from hospital records, police reports or ambulance intervention reports. Accident data should be obtained for a minimum of one year. The other critical information that is needed exposure data in the form of traffic flow through intersections, typically the annual average daily traffic (AADT) of the major and minor intersecting streets. The accident and traffic flow datasets will be used to select sample intersections for further analysis.

The next step is to prepare the accident and flow data according to the needs of this project. If neither of these datasets is available, the intersection sample for data collection will be selected randomly.

\section{Data Preparation Process}

First, the accident data must be filtered to include only the accidents that occurred at night time. Sunset and sunrise times do not fully represent dark conditions since the sky is not completely dark for some time after sunset and before sunrise. Alternatively, twilight times are used. Based on the Mariam-Webster dictionary, twilight is the "light from the sky between full night and sunrise or between sunset and full night produced by diffusion of sunlight through the atmosphere and its dust". Using this definition, night time is considered as the time when evening twilight ends until the time when morning twilight starts. If twilight times are not available, a thirty minute interval after sunset and before sunrise can be used.

The second step is to plot all the night time accidents in a geographic mapping and analysis software such as ArcGIS. Accidents occurring in a $15 \mathrm{~m}$ radius from an intersection are associated with it using a circular buffer and a spatial joint. The $15 \mathrm{~m}$ buffer from the center of the intersection was chosen as the effective area to analyse the night time light measures. It is a result of a sensitivity analysis for four different buffer radiuses, 5, 10, 15 and $20 \mathrm{~m}$. Finally, the intersections with flow data and night time accidents constitute the candidate set from which a sample is selected for field data collection.

\section{Sample Selection}

There are two methods to select a sample for data collection, either randomly or by using intersection accident and flow data. If these datasets are not available, or there is a time constraint to perform a light audit where no accident and flow data can be collected, sample intersections for the lighting audit can be selected randomly throughout the city. This random selection must cover different districts within the city and must have variability in the type of roads crossing at the intersection, e.g. arterial-collector, arterial-local, etc. 
The other method is to use the data prepared in the previous step to select intersections for data collection. The sample intersections are selected by identifying hotspots based on the number of accidents and the flow through the intersection.

The accident risk level at intersections is estimated using the Empirical Bayes (EB) approach (34). For the statistical analysis, we start by assuming that for each site $i$, the number of accidents over a period of time $\left(Y_{i}\right)$ follows a Poisson distribution, where $\theta_{i}$ is the mean accident frequency and follows a Gamma distribution, i.e., $Y_{i} \mid \theta_{i} \sim$ Poisson $\theta_{i}$ and $\theta_{i} \sim \operatorname{Gamma} \varphi, \frac{\varphi}{\mu_{i}}$. According to this popular Poisson/Gamma model, the conditional probability $p\left(\theta_{i} \mid y_{i}\right)$ is also Gamma distributed with shape $a=\left(y_{i}+\varphi\right)$ and scale parameter $b=$ $\left(1+\frac{\varphi}{\mu_{i}}\right)$. From this, the popular EB estimator is given by the posterior mean of $\theta_{i}$ :

$$
\begin{gathered}
E\left(\theta_{i} y_{i}=\frac{y_{i}+\varphi}{1+\varphi / \mu_{i}} \text { or } E B_{i}=E\left(\theta_{i} y_{i}=1-w_{i} y_{i}+w_{i} \mu_{i}\right.\right. \\
w_{i}=\frac{\varphi}{\varphi+\mu_{i}} \\
\mu_{i}=\beta_{0} F_{1 i} \beta_{1} F_{2 i} \beta_{2}
\end{gathered}
$$

Where: $F_{1 i}-$ flow in the major approach at intersection $\mathrm{i}$

$F_{2 i}$ - flow in the minor approach at intersection i

$\beta$ - regression coefficients obtained from the data

$\mu_{i}$ - safety performance function depending on site-specific factors

$\varphi$ - dispersion parameter

Using the number of accidents for each intersection as well as the flow in the major and minor approaches, a negative binomial regression model is run using a statistical analysis program such as Stata. From there, the regression coefficients and the dispersion parameter are used in the formula to obtain the safety performance function and EB. Then, the potential improvement factor, called risk thereafter, is calculated as follows:

$$
P I_{i}=E B_{i}-E B_{r p}
$$

where $E B_{r p}$ is the average number of night time accidents in the reference population. Based on the PI results, arbitrary thresholds are used to indicate high-risk, medium-risk, low-risk and PI values below zero can be considered as safe intersections.

\section{Data Collection Procedure on Sample Intersections}

\section{Equipment Used for Data Collection}

Skye Instruments Ltd (35) manufactures light measurement sensors and data loggers. For this project, the SpectroSense2+ (SKL 925) logging meter was used. The SKL 925 has the option of recording measurement position via a GPS receiver. The sensors (SKP 218) manufactured by the same company are two one channel sensors. The sensors have a photodiode detector responsive to wavelengths from 280 to $1100 \mathrm{~nm}$ which includes the visible light wavelengths. The sensors measure illuminance levels in units of kilo-lux.

For data collection purposes, these sensors should be attached to a stable handle in a way that one sensor is facing up and the other is facing down. The sensor facing up is collecting data from the sources of the light representing the ambient light perceived by the eyes. The sensor facing down indicates how bright the road surface is, measuring the road surface reflectance: this

sensor is not used in the present study as no requirement for lighting is based on it. The following is the list of what is needed for the data collection:

\section{- Data logger}


- Photometric sensors

- Data collection sheets with pen or pencil

- Construction vest

\section{Sample Data Collection Process}

Since the data collection process happens during the night, and some intersections may be located in unsafe areas, there should be at least two people collecting data. The twilight time for each evening should be checked and data collection should start after the evening twilight ends. Safety vests should be worn in order to be clearly visible to drivers.

Before starting, the logger must be checked for battery level and memory space. The light measurement interval is selected to be $1 \mathrm{~s}$. The SKL 925 logger records the date, time, illuminance level from both sensors, and GPS coordinates. One of the problems with analysing the data from the logger is that in urban areas, the GPS does not give accurate readings because of the urban canyon effect, especially when the distance traveled is only from one side of the street to the other. In order to overcome this problem, before starting to cross the street with the logger, the time and location of crossing is recorded. In this way, when retrieving the data, the illuminance measurement from each crossing has a unique start time and intersection name.

At signalised intersections, since the data collector starts at a distance away from the intersection, roughly $15 \mathrm{~m}$ as shown in Figure 1, and data logging should happen at relatively constant speed, there should be enough time to cross the intersection. To avoid stopping behind red lights when logging data, the data collector starts logging just when the traffic signal in their direction turns green so that there is enough time to reach the intersection and cross with a constant speed while the light is still green. For non-signalised intersections, the same procedure is followed without waiting for the traffic lights.

Using the mentioned sensors and logger, several tests were performed for sensitivity analysis. Initially, data was collected at a single intersection in different weather conditions to check for illuminance variability according to different weather conditions (clear sky, mainly clear, overcast, after rain, snow on the ground). Results showed no correlation between the two factors, meaning that the variation of light measurements were not dependent on the weather. The second test was performed using a pole with the sensors attached to it. Data was collected with different pole heights ranging from $80 \mathrm{~cm}$ to $260 \mathrm{~cm}$. The results did not show much variability with regards to height. Therefore, for the data collection, the data collector can collect data holding the sensors at any convenient height. For each night of the data collection, the temperature, sky condition and moon phase are recorded for further analysis.

While the illuminance data is being collected, the accompanying data collector fills in a data collection sheet (Figure 1). The data collection sheet aims to gather information on the type of intersection, location of light poles, locations of trees which may block light, location of commercial light (defined as the light coming from stores, restaurants and other roadside buildings), built environment characteristics and any other notes about the intersection.

\section{Field Data Analysis}

At the end of each data collection, the illuminance measurements from the sensor should be downloaded from the logger using the SpectroSense2+ software. The average illuminance values for each approach of the intersection are calculated using their corresponding start times. The average illuminance of the four approaches is taken as the average illuminance of the intersection. Then, the uniformity ratio of the approaches is calculated. Table 1 and Figure 3 in 
the case study section represent the average illuminance calculated for each approach of an intersection and the raw measurements from the sensors respectively.

\section{MONTRÉAL CASE STUDY}

\section{Study Area}

The case study for applying the mentioned methodology is in the Island of Montréal, Québec, Canada. The Island of Montréal has two different types of lighting specifications provided by the Ministère des Transports du Québec, and lighting provided by local municipalities (4). The former uses IES standards and the latter uses TAC standards for road lighting. Given the two different standards, the problem of inconsistent lighting arises throughout the city, especially at intersections (4).

\section{Data Source, Sample Selection and Data Collection}

Accident data for this project was obtained from Montréal police reports from 2001 to the end of 2010. This data included the vehicle-vehicle and pedestrian-vehicle accidents, their location in latitude and longitude coordinates. Bicycle accident data is not used in this study because night time bicycle accidents are under-reported and the flow of bicycles during night time is very low.

The intersection geometry and traffic flows were acquired from data collected manually by the McGill University transportation engineering group in 2008-2009. These manual counts were done during 8 hours and used to determine AADT for vehicles. Counts were taken during the two peak periods ( 3 hours each) and 2 hours during the noon period. Expansion factors considering weekly, monthly and the 24 hours of the day were used to extrapolate counts. Here it is assumed that flow intensity during the day is proportional to the night period; therefore AADT is still used as a measure of traffic activity during night time. This intersection inventory includes the intersection ID, names of intersecting streets, latitude and longitude coordinates of the intersection point, the AADT flow for each approach and the road type (national, arterial, collector, and local).

These two datasets, accident and intersection flow, were used to select a sample of intersections with a wide range of accident frequency and land use variability, which are discussed in the following sections.

\section{Accident Data Preparation}

From the Montréal accident data, twilight times were used for each day of the year to filter through the 10 years of accident data and select only accidents that occurred during night time. Based on this approach, 12,433 accidents occurred at night time, which accounts for approximately $19 \%$ of the total vehicle-vehicle and pedestrian-vehicle accidents recorded in Montréal throughout the 10 years. Accidents that occurred within a $15 \mathrm{~m}$ radius of an intersection were then selected using ArcGIS.

\section{Selecting Intersection Sample}

Using the EB approach, intersections with night time accidents were ranked based on their accident risk level and a random sample of intersections were selected for data collection. Based on the potential improvement values, risk thresholds were defined as follows:

- $\quad$ PI $>7$ as High-risk

- $2<$ PI $<7$ as Medium-risk

- $0<\mathrm{PI}<2$ as Low-risk 
- $\quad \mathrm{PI}<0$ as No-risk

Factors considered for selecting the intersection sample were the number of night time accidents per intersection and AADT flow for major and minor approaches. A total of 85 intersections were randomly selected within all the accident risk categories, from high to low accident risk, including intersections without any accident, as shown in Figure 2. These intersections were selected throughout the city covering the downtown and suburban areas. Different types of roads with different land use were selected in different districts. Of the selected intersections, $26 \%$ intersections were high-risk, $33 \%$ were medium-risk, $28 \%$ were low-risk and $13 \%$ were no risk intersections as shown in Figure 2.

\section{Data Collection on Sample Intersections}

Data was collected for the selected intersections in June and July 2013, from the end of the evening twilight before the morning twilight. For each night of the data collection, the temperature, weather and moon phase was recorded from Environment Canada (36). The data was collected using the procedure described in the previous section.

\section{Sample Descriptive Analysis and Model}

This section covers the analysis of the illuminance of the intersection sample and of the night time accidents, and the effects of exogenous variables on them.

From the lighting point of view, some indicators such as the average illuminance of each approach, the average illuminance for the whole intersection and the uniformity values were compiled. The uniformity ratio for each intersection is calculated using the average intersection illuminance over the minimum average illuminance of the four approaches of the intersection. The average illuminance value and uniformity ratio of each intersection are compared with the lighting standards. Table 1 illustrates the average illuminance measured for each approach of one of the intersection samples. Figure 3 presents the point measurements collected by the sensor for the same intersection. In other words, this figure illustrates the amount of illuminance of each point in each direction. Comparing the information in Table 1 and Figure 3 shows that east side of the intersection is brighter at night than the west side.

For this intersection, the average intersection illuminance is 14.7 lux and the uniformity ratio is 1.7. Comparing these values with the arterial-local intersection lighting standards, where the average illuminance must be above 19 lux and uniformity ratio should be below 3, indicates that this intersection is not lit according to standard, but the uniformity ratio meets the standards.

Accordingly, the average illuminance and the uniformity ratio of the 85 selected sample intersections are compiled based on the collected point illuminance measurements of each intersection. The analysis of average illuminance indicates that around $60 \%$ of the intersections are below road lighting standards. This can be studied for each intersection along with their level of risk. Figure 4 shows the distribution of intersection with standard and non-standard lighting over their level of risk. The distributions are very similar and show an absence of a visible link between safety and whether an intersection meets the illuminance standard or not.

Figure 5 shows a sample of four types of road intersections depending on the types of roads. In these diagrams, the points below the red dotted line are those that do not meet the city specification standards for road lighting. The four plots comprise 49 intersections among the 85 samples intersections. It seems again that, even if the sample is stratified by road types at the intersection, there does not seem to be a strong relationship between the average illuminance and safety as measured by PI, or between substandard lighting and risk levels. 


\section{Model Results}

To analyse the effect of night time accident and average illuminance on each other and on other built environment indicators, the following set of binary and numerical variables were used:

- Night accidents: raw number of night time accidents, and PI value

- Illuminance: average intersection illuminance, maximum and minimum average illuminance of the four approaches of each intersection, sub-standard illuminance indicator, and uniformity ratio

- Traffic: ratio of minor to major flow

- Temperature

- Weather: clear sky, mainly clear sky, few clouds, cloudy sky

- Moon: more or less than half full

- Hour of night for the data collection: 9pm-10pm, 10pm-11pm, 11pm-12am, 12am-1am

- Built environment: number of approaches with commercial light present, number of approaches with trees, number of approaches with light pole present

- Land use: number of sides with commercial land use, residential land use, industrial land use, parks parking lots, and gas stations

- Road type: national, arterial, collector, local

The effects of each of these variables were measured on the number of night accidents and illuminance. To model the number of night accidents, a negative binomial regression is used since accident is a count variable. For the illuminance model, a linear regression model is used since illuminance is a continuous variable. Different combinations of these variables were added to obtain the best fitted model.

The results for significant indicators affecting the number of night time accidents are presented in Table 2. Variables that have an effect on night time accidents are traffic and illuminance. The coefficients presented in Table 2 show that traffic increases the chance of night time accidents. Since the ratio of minor to major flow is included in the model, the number of night time accidents will increase when minor flow increases and gets close to the major flow. Intersections with sub-standard illuminance also increase the chance of night time accidents.

Model results for indicators affecting illuminance are presented in Table 2. The significant variables were weather, hour of night, and built environment. The effect of the weather indicator was negative meaning that clear sky reduces average intersection illuminance. This may be due to the fact that the presence of clouds captures and reflects the light from the environment, whereas clear skies do not have that effect. The hour of night indicator has a positive effect on illuminance. Based on the model results, the average intersection illuminance is increased after midnight. The reason for this effect is not known. The built environment variables that came out significant are the number of approaches with commercial light and number of approaches with light poles. This is reasonable since the average intersection illuminance would increase if there are more commercial lights and light poles present at the intersection.

\section{CONCLUSION}

This paper proposed a methodology for the audit of road lighting and safety. The methodology applied to the Island of Montréal showed that from the sample of 85 intersections, $59 \%$ had substandard lighting. Statistical results showed that sub-standard average intersection illuminance increases the chance of night time accidents, and minimum to maximum traffic flow ratio also increases night time accidents. It also showed that average illuminance increases with the 
presence of light poles and commercial light, and after midnight. Average illuminance is decreased when the sky is clear. This study points at a relationship between road lighting and safety and highlights the need for more data collection and analysis.

A limitation of this project is the use of 10 years of crash data. This was necessary due to the low number of crash occurrences at the studied intersections. The assumption is that road lighting changes throughout these years was limited.

Future work in this field can be done to improve and expand the findings in this paper, such as increasing the sample size and considering more variables. Further work can also make use of the road surface reflectance which is collected from a sensor facing down indicating how bright the road surface is. It will also include traffic flow counts during night time instead of using the AADT for night time traffic flow. 


\section{ACKNOWLEDGMENTS}

The authors wish to acknowledge the financial support from the Road Safety Research Program funded by the Fonds de recherche du Québec - Nature et technologies (FQRNT), the Québec Ministry of Transportation and the Fonds de recherche du Québec - Santé (FRQS), as well as the help from the City of Montréal and boroughs that authorized the data collection.

\section{REFERENCES}

1. Bullough, J. D., E. T. Donnell, and M. S. Rea. To Illuminate or Not to Illuminate: Roadway Lighting as it Affects Traffic Safety at Intersections. Accident Analysis and Prevention, Vol. 53, 2013, pp. 65-77.

2. Bullough, J. D., N. P. Skinner, and M. S. Rea. Design and Evaluation of Effective Crosswalk Illumination. Publication FHWA-NJDOT-2009-003. FHWA, U.S. Department of Transportation, 2009.

3. Beyer, F. R., and K. Ker. Street Lighting for Preventing Road Traffic Injuries. Cochrane Database of Systematic Reviews, Issue 1, Art. No. CD004728, 2010.

4. Bruneau, J. F., and D. Morin. Standard and Nonstandard Roadway Lighting Compared with Darkness at Rural Intersections. In Transportation Research Record: Journal of the Transportation Research Board, No. 1918, Transportation Research Board of the National Academies, Washington, D.C., 2005, pp. 116-122.

5. Ekrias, A., M. Elohoma, L. Halonen, X. J. Song, X. Zhang, and Y. Wen. Road Lighting and Headlights- Luminance Measurements and Automobile Lighting Simulations. Building and Environment, Vol. 43, 2008, pp. 530-536.

6. DiLaura, D., K. Houser, R. Mistrick, and G. Steffy. The Lighting Handbook. Illumination Engineering Society, New York, 2000.

7. Road Lighting as an Accident Countermeasure. CIE Pub-lication. No. 93, Central Bureau of the CIE, Vienna, 1992.

8. Bouchard, J., et al. Transportation SafetyPolicy 2001-2005: Road Component. Ministère des Transports du Québec, Québec, 2005.

9. Ward, H., N. Shepherd, S. Robertson, and M. Thomas. Night Time Accidents. Report to The AA Motoring Trust and Rees Jeffreys Road Fund, University College London, 2005.

10. Hasson, P., and P. Lutkevich. Roadway Lighting Revisited. Public Roads, May/June 2002.

11. Yannis, G., A. Kondyli, and N. Mitzalis. Effect of Lighting on Frequency and Severity of Road Accidents. Proceeding of the Institution of Civil Engineering, Vol. 166, 2012, pp. 271-281. 
12. Wanvik, P. O. Effects of Road Lighting on Motorways. Traffic Injury Prevention, Vol. 10 (3), 2009, pp. 279-89.

13. Donnell, E. T., R. J. Porter, and V. N. Shankar. A Framework for Estimating the Safety Effects of Roadway Lighting at Intersections. Safety Science, Vol. 48, 2010, pp. 14361444.

14. Transport Quebec. Road safety and road lighting. Gouvernment du Quebec. http://www1.mtq.gouv.qc.ca/en/projet_recherche/description.asp?NO_PROJ=I117.1P1. Accessed Jun. 10, 2013.

15. The Royal Society for the Prevention of Accidents. Street Lighting and Road Safety. Road safety information: Highway, Birmingham UK, 2009.

16. Isebrands, H., et al. Safety Impacts Of Street Lighting at Isolated Rural Intersections. Center for Transportation Research and Education Iowa State University, Ames, IA, 2004.

17. Assum, T., T. Bjørnskau, S. Fosser, and F. Sagberg. Risk Compensation - the Case of Road Lighting. Accident Analysisand Prevention, Vol. 31, 1999, pp. 545-533.

18. Goodman, T., A. Forbes, H. Walkey, et al. Mesopic Visual Efficiency IV: a Model with Relevance to Nighttime Driving and Other Applications. Lighting Research and Technology, Vol. 39, 2007, pp. 365-392.

19. Griffith, M. S. Comparison of the Safety of Lighting Option on Urban Freeways. Public Roads, Vol. 58, 1994, pp. 8-15.

20. Jackett, M., and W. Frith. Quantifying the Impact of Road Lighting on Road Safety - A New Zealand Study. IATSS Research, Vol. 36, 2013, pp. 139-145.

21. Gonzalez-Velez, E. Safety Evaluation of Roadway Lightin Illuminance Levels and its Relationship with Nighttime Crash Injury Severity for West Central Florida Region. Ph.D. Dessertation, University of South Florida, 2011.

22. Transport Canada. Road Safety in Canada. Gouvernment of Canada. http://www.tc.gc.ca/eng/roadsafety/tp-tp15145-1201.htm. Accessed Jun. 10, 2013.

23. London Department for Transportation. Manual for Streets. Thomas Telford Publishing, London, UK, 2007.

24. Green, E. R., K. R. Agent, M. L. Barrett, and J. G. Pigman. Roadway Lighting and Driver Safety. Publication KTC-03-12/SPR247-02-1F, Kentucky Transportation Center, 2003.

25. McLean, D., I. Lwein, and P. Lutkevich. Guide for the Design of Roadway Lighting. Transportation Association of Canada (TAC), 2006. 
26. Transport Canada. Intersection-Related Crashes in Canada: Driver Characteristics and Casualty Trends. Road Safety Directorate Transport Canada, 2007.

27. Rea, M. S., J. D. Bullough, C. R. Fay, J. A. Brons, J. V. Derlofske, and E. T. Donnell. Review of the Safety Benefits and other Effects of Roadway Lighting. Prepared for National Cooperative Highway Research Program Transportation Research Board of The National Academies Lighting Research Center, 2009.

28. Bullough, J. D., M. S. Rea, and Y. Zhou. Analysis of Visual Performance Benefits from Roadway Lighting. Prepared for National Cooperative Highway Research Program Transportation Research Board of The National Academies Lighting Research Center, 2009.

29. Lightin Research Center. Recommendations for Evaluating Street and Roadway Luminaires. Alliance for Solid-State Illumination Systems and Technologies, Vol. 10, 2011.

30. Schieber, F. Age and Glare Recovery Time for Low-Contrast Stimuli. Department of Psychology, University of South Dakota, Vermillion, 1994.

31. Box, P. C. Relationship Between Illumination and Freeway Accidents. IERI Project 85-67 Illuminating Research Institute, New York April, 1970, pp. 1-83.

32. Schreuder, D. A. The Relationship Between the Level of Street Lighting and Traffic Safety. SOV Institute for Road Safety Research, Netherlands, 1989.

33. Schreuder, D. A. Public Lighting: The Effectiveness for Road Transport. Proceedings of the Institution of Public Lighting Enigneers IPLE Diamond Jubilee Conference, 1984, pp. 67-72.

34. Miranda-Moreno, L. F., A. Labbe, and L. Fu. Bayesian Multiple Testing Procedures for Hotspot Identification. Accident Analysis and Prevention, Vol. 39, 2007, pp. 1192-201.

35. Skye Instruments Ltd. http://www.skyeinstruments.com/category/products/. Accessed Feb.1, 2013.

36. Environment Canada. Montréal, QC - 7 Day Forecast. Gouvernment of Canada. http://www.tc.gc.ca/eng/roadsafety/tp-tp15145-1201.htm. 


\section{LIST OF FIGURES AND TABLES}

FIGURE 1 Data collection sheet.

FIGURE 2 Map of selected intersections and their risk.

FIGURE 3 Map the collected illuminance measurements at a sample intersection.

FIGURE 4 Risk distributions among intersections with standard and non-standard lighting.

FIGURE 5 Average intersection illuminance as a function of PI. $14 \%$ of the intersections have 2 arterials, $18 \%$ have an arterial and a collector, $14 \%$ arterial and a national and $12 \%$ a collector and a national. $42 \%$ of the intersections are not represented in these plots.

TABLE 1 Average Illuminance of Each Approach in a Sample Intersection

TABLE 2 Effects of Exogenous Variables on Night time Accidents and Average Illuminance 
Draw the location of light poles $\mathrm{L}$ and the location of trees $\mathrm{T}$

\section{Intersection Name:}

\section{Date:}
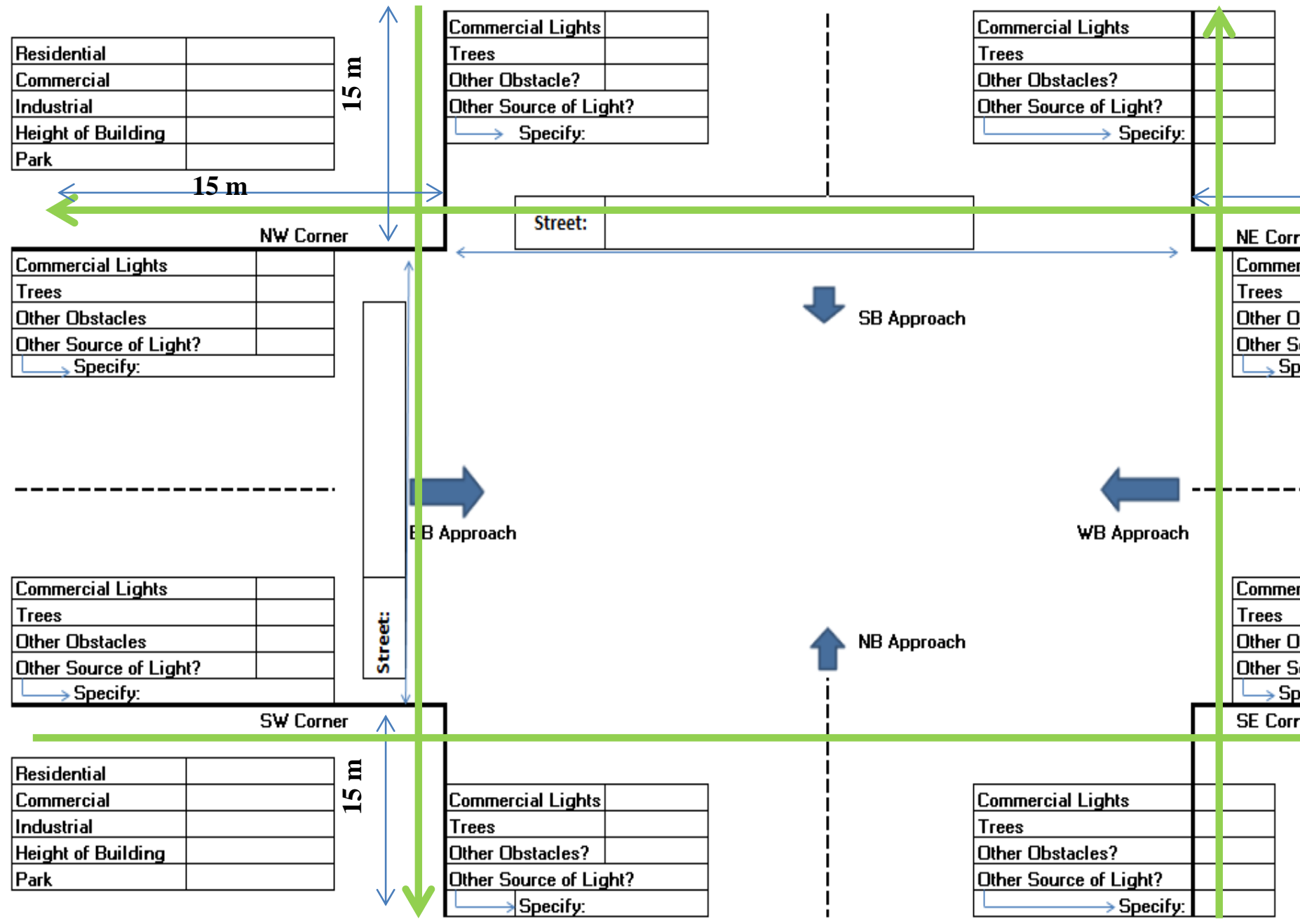

\begin{tabular}{|l|l|}
\hline Residential & \\
\hline Commercial & \\
\hline Industrial & \\
\hline Height of Building & \\
\hline Park & \\
\hline
\end{tabular}

15

LE Corner

SB Approach

Commercial Lights

Trees

Other Obstacles

Other Source of Light?

$\longrightarrow$ Specify:

FIGURE 1 Data collection sheet. 


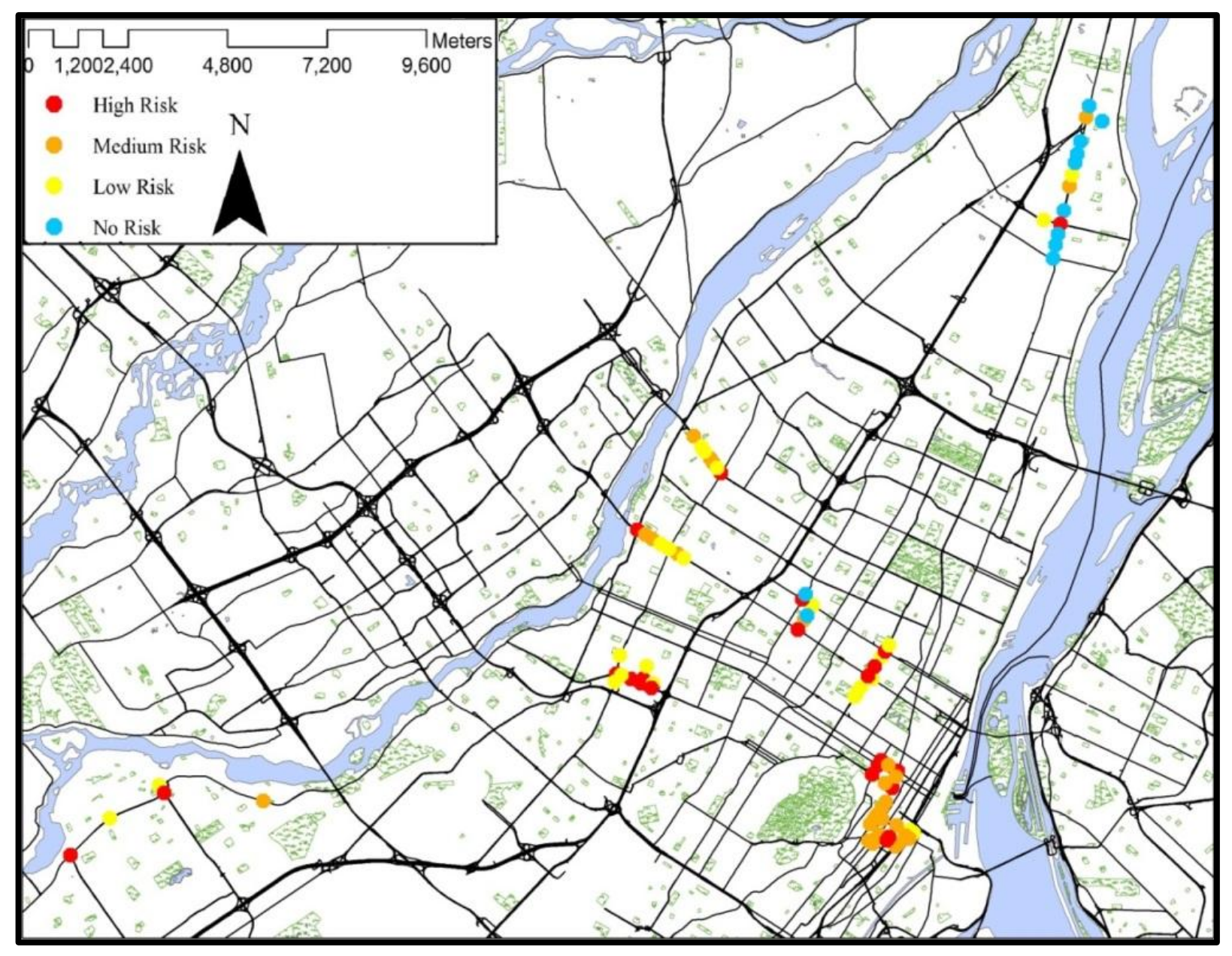

FIGURE 2 Map of selected intersections and their risk. 


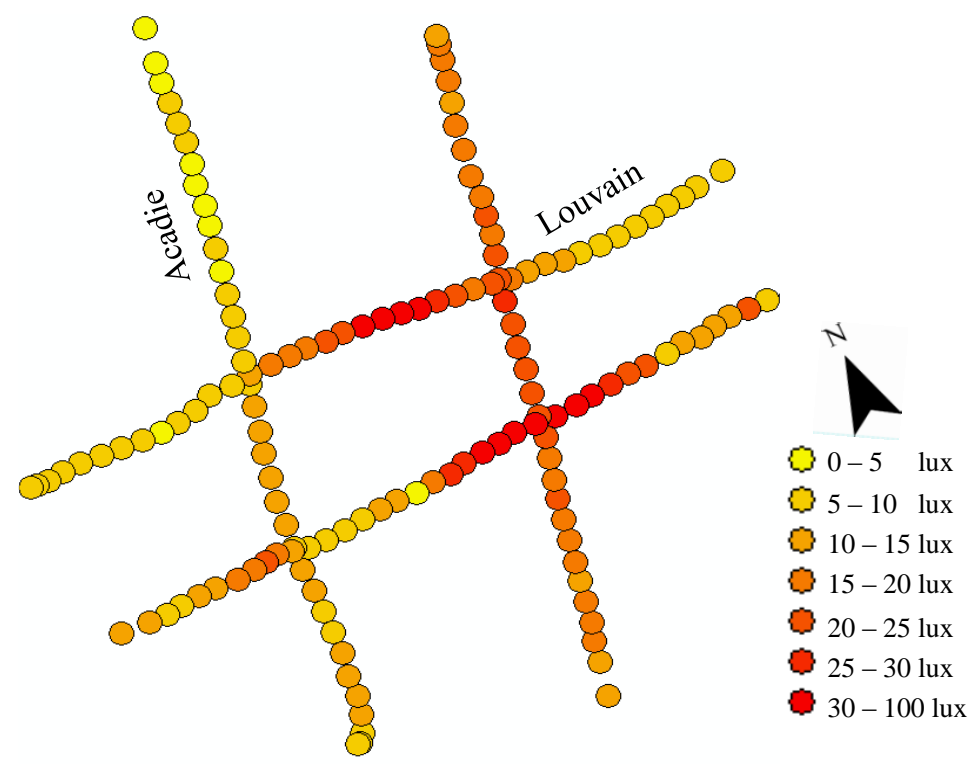

FIGURE 3 Map the collected illuminance measurements at a sample intersection. 


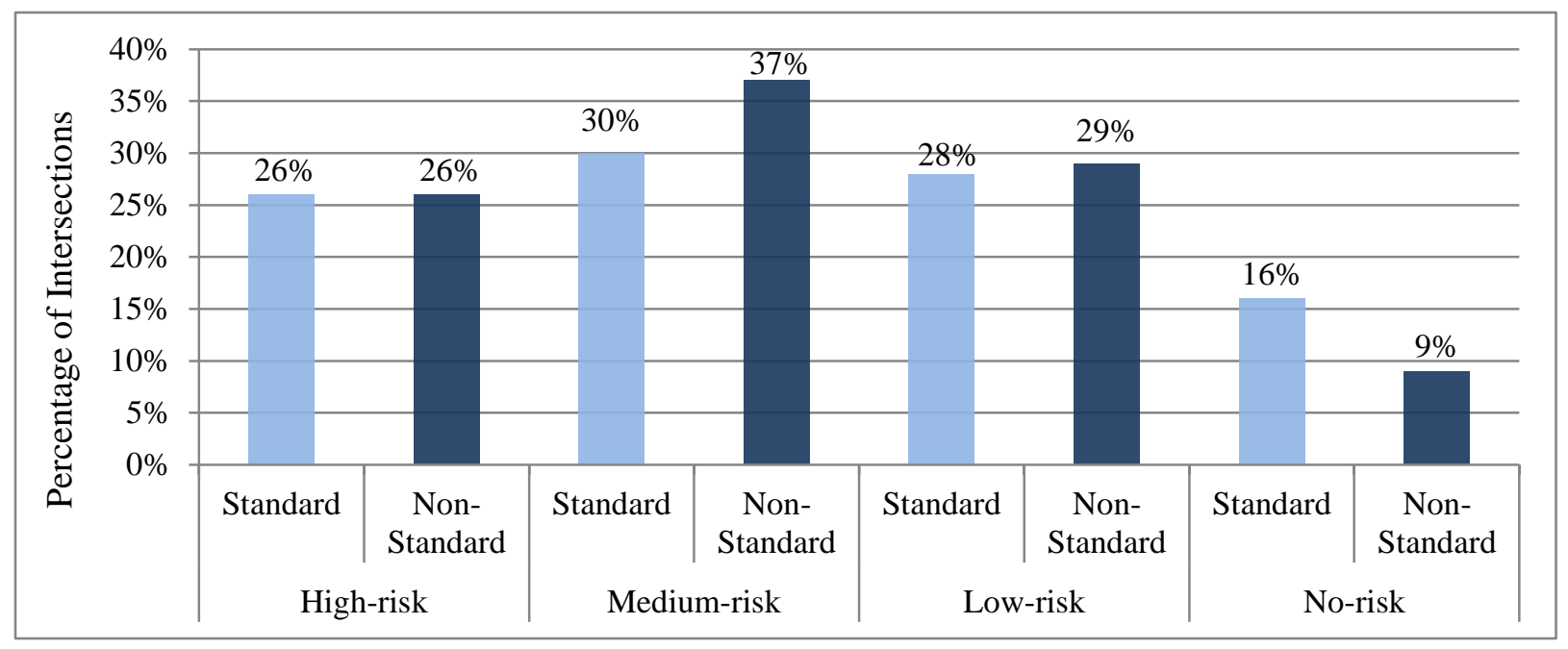

FIGURE 4 Risk distributions among intersections with standard and non-standard lighting. 


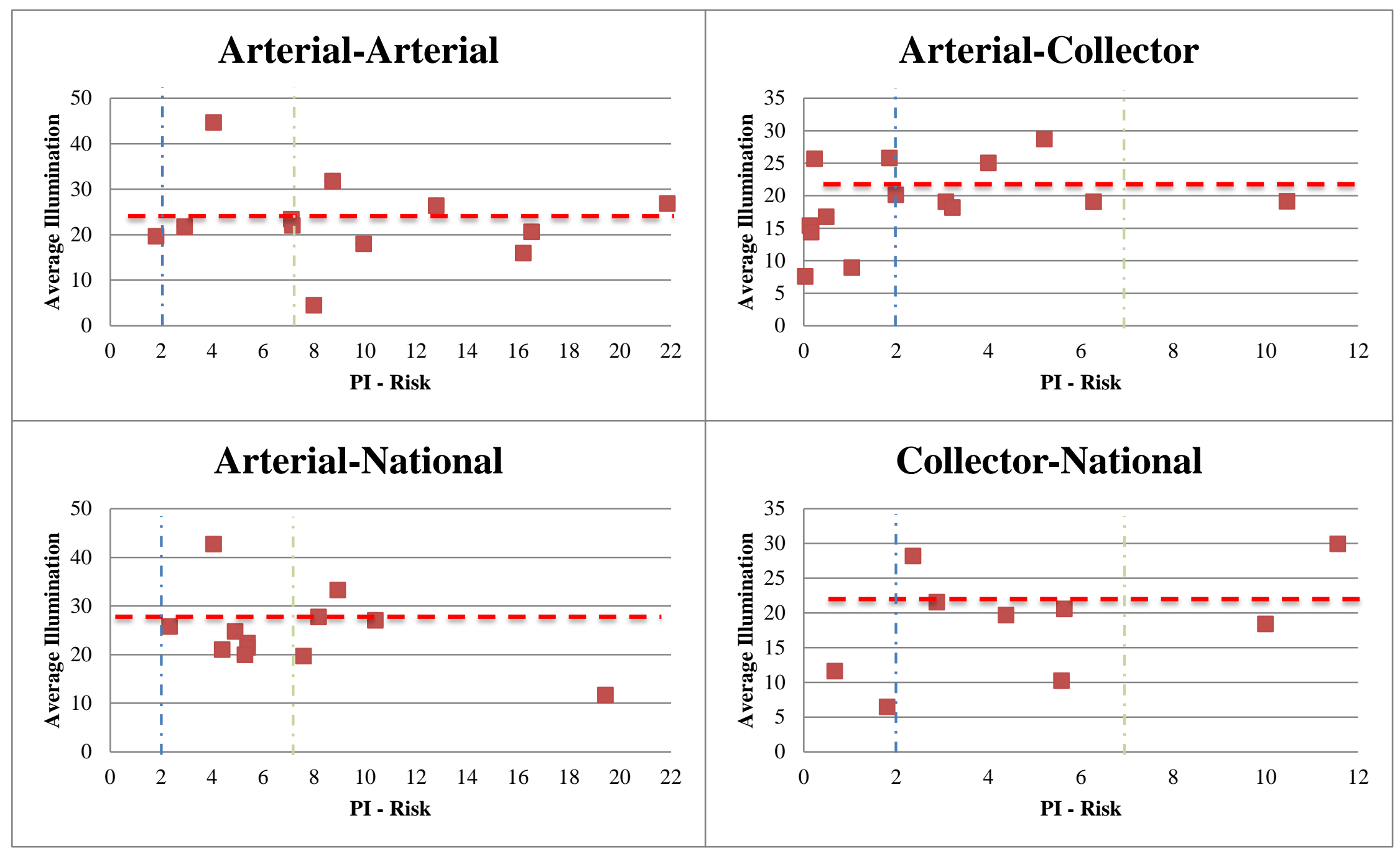

Lighting standard threshold ---

High-risk threshold

Medium-risk threshold

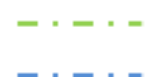

FIGURE 5 Average intersection illuminance as a function of PI. $14 \%$ of the intersections have 2 arterials, $18 \%$ have an arterial and a collector, 14 $\%$ arterial and a national and $12 \%$ a collector and a national. $42 \%$ of the intersections are not represented in these plots. 
TABLE 1 Average Illuminance of Each Approach in a Sample Intersection

\begin{tabular}{||l||l|l|l|l|l||}
\hline ID & Date & Street name & Side & Direction & Average Illuminance (lux) \\
\hline 1246 & 08 -Jul-13 & Louvain E (Local) & North & E to W & 17.5 \\
\hline 1246 & 08 -Jul-13 & Acadie (Arterial) & West & N to S & 8.5 \\
\hline 1246 & 08 -Jul-13 & Louvain E (Local) & South & W to E & 14.0 \\
\hline 1246 & 08 -Jul-13 & Acadie (Arterial) & East & S to N & 18.8 \\
\hline
\end{tabular}


TABLE 2 Effects of Exogenous Variables on Night time Accidents and Average Illuminance

\begin{tabular}{|c|c|c|c|c|c|c|}
\hline \multicolumn{2}{|c|}{ Number of night accidents } & Coefficient & z-value & p-value & \multicolumn{2}{|c|}{ [95\% Conf. Interval] } \\
\hline Traffic & ratio minor to major flow & 0.91 & 3.23 & 0.00 & 0.36 & 1.46 \\
\hline Illuminance & substandard illuminance & -0.26 & -1.60 & 0.11 & -0.58 & 0.06 \\
\hline \multicolumn{2}{|l|}{ Constants } & 1.60 & 10.10 & 0.00 & 1.29 & 1.91 \\
\hline \multicolumn{2}{|c|}{ Average illuminance } & Coefficient & z-value & p-value & \multicolumn{2}{|c|}{ [95\% Conf. Interval] } \\
\hline Weather & clear sky & -4.28 & -2.60 & 0.01 & -7.56 & -1.00 \\
\hline Hour of night & time $12 \mathrm{am}-1 \mathrm{am}$ & 7.96 & 2.75 & 0.01 & 2.21 & 13.72 \\
\hline \multirow{2}{*}{$\begin{array}{l}\text { Built } \\
\text { Environment }\end{array}$} & $\begin{array}{l}\text { number of approaches } \\
\text { with commercial light }\end{array}$ & 0.91 & 2.28 & 0.03 & 0.12 & 1.69 \\
\hline & $\begin{array}{l}\text { number of approaches } \\
\text { with light pole }\end{array}$ & 1.66 & 3.86 & 0.00 & 0.80 & 2.52 \\
\hline \multicolumn{2}{|l|}{ Constants } & 11.82 & 5.15 & 0.00 & 7.25 & 16.39 \\
\hline
\end{tabular}

